\title{
Faraday Rotation Measure Observations on the Pc-Scale: Prob- ing the AGN Environment
}

\section{G. B. Taylor}

National Radio Astronomy Observatory, Socorro, NM 87801, U.S.A.

T. Venturi

\section{Istituto di Radioastronomia CNR, Bologna, Italy}

P. S. Udomprasert ${ }^{1}$

Princeton University, Princeton, NJ 08544, U.S.A.

\begin{abstract}
We present multi-frequency VLBA polarimetric observations of a small sample of quasars revealing their Faraday rotation measure (RM) structure at $\sim 1$ mas resolution. Two of the sources (OQ 172 and 3C 216) were chosen on the basis of high RMs seen by the VLA and single dish telescopes. A few more typical quasars (3C 273, 3C 279, 3C 345 and $3 \mathrm{C} 380$ ) were also included in the study. In OQ $172,3 \mathrm{C} 273,3 \mathrm{C} 279$ and $3 \mathrm{C} 380$ we find high $\mathrm{RMs}(>3000$ $\mathrm{rad} \mathrm{\textrm {m } ^ { - 2 }}$ ) associated with the nuclear region. Only 1-10 mas from the nucleus the jet $|\mathrm{RM}| \mathrm{s}$ in all 4 of these sources fall to less than $100 \mathrm{rad} \mathrm{m}^{-2}$. This RM structure may be produced by strong magnetic fields in the the narrow line region of the quasar.
\end{abstract}

Typical extragalactic radio sources, like 3C 279 and 3C 380, display Faraday rotation measures (RMs), of $10-100 \mathrm{rad} \mathrm{m}^{-2}$ at arcsec resolution. A small group of $\sim 20$ sources has been found to have RMs exceeding $1000 \mathrm{rad} \mathrm{m}^{-2}$ (Inoue et al. 1995; Taylor, Inoue, \& Tabara 1992). About half of these are compact steep spectrum (CSS) sources. Those of larger angular extent, for example, Hydra A and Cygnus A, have been observed with the VLA and have been shown to possess ordered RM structure on scales of 1-100 kpc (Taylor \& Perley 1993; Dreher, Carilli, \& Perley 1987). A good candidate mechanism for producing such high RMs is a magnetized X-ray emitting cluster gas with field strengths of $\sim 10 \mu \mathrm{G}$.

In the case of the high RM CSS sources, observations at a resolution of 1 arcsec or more were insufficient to measure the scale of the magnetic fields and thereby determine their origin. Thus we performed VLBA observations of the CSS quasars OQ 172 and 3C 216. Very high RMs (in excess of $40,000 \mathrm{rad} \mathrm{m}^{-2}$ ) were found in the nuclear region of OQ 172 (Udomprasert et al. 1997). These high RMs cause substantial depolarization at low frequencies and it occurred to us that the low polarization seen in many quasar cores at $6 \mathrm{~cm}$ (Cawthorne et al. 1993) may be the result of depolarization from a Faraday screen. Thus we also performed multi-frequency VLBA polarimetry on four well studied quasars: 3C 273, 3C 279, 3C 345, and 3C 380 (see the paper by Polatidis \& Wilkinson in these Proceedings, p. 77).

VLBA observations of $3 \mathrm{C} 216$ at 5 and $8 \mathrm{GHz}$ reveal high $\mathrm{RMs}$ concentrated in a bowshock where the jet bends through $90^{\circ}$. These RMs are consistent with VLA observations (Taylor, Ge, \& O'Dea 1995), and could be produced by interactions with a cluster medium.

In contrast, the VLBA observations at 8,12 and $15 \mathrm{GHz}$ of $3 \mathrm{C} 273,3 \mathrm{C} 279$ (see Fig. 1), and 3C 380 reveal rest frame RMs of $2700,-3100$, and $-2900 \mathrm{rad}$ $\mathrm{m}^{-2}$ in the nuclear region. Between 1 and 10 mas from the core the jet $|\mathrm{RM}| \mathrm{s}$

\footnotetext{
${ }^{1}$ Present address: Caltech, 105-24, Pasadena, CA 91125, U.S.A.
} 
fall to less than $100 \mathrm{rad} \mathrm{m}^{-2}$, small enough to be produced by the passage of the radiation through the interstellar medium of our galaxy.

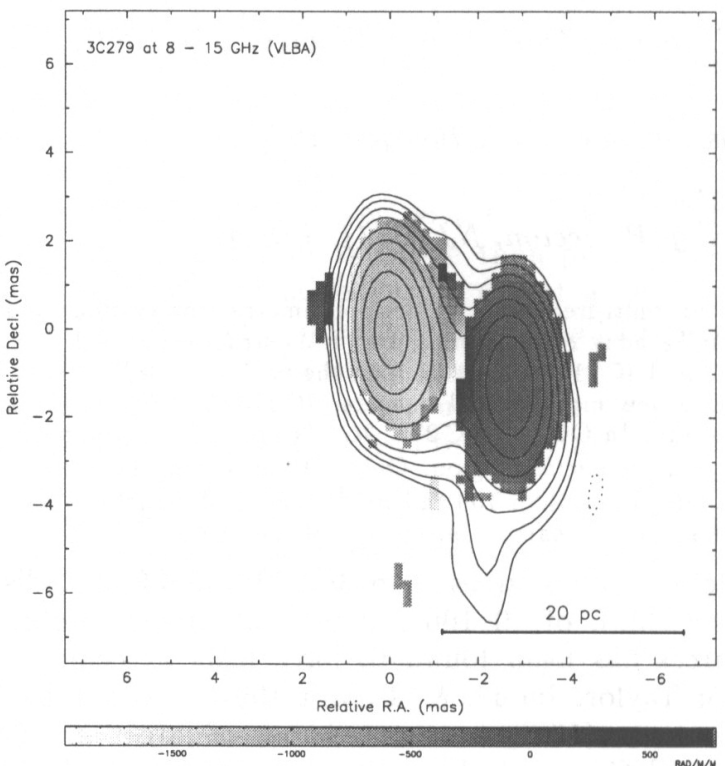

Figure 1. A map of the Faraday rotation measures in $3 \mathrm{C} 279$ with contours from the total intensity image at $8 \mathrm{GHz}$ overlaid. The grey scale range is from -2000 to $700 \mathrm{rad} \mathrm{m} \mathrm{m}^{-2}$. The core has a RM of $-1300 \pm 50 \mathrm{rad} \mathrm{m}^{-2}$ while the jet has a RM of $-60 \pm 50 \mathrm{rad} \mathrm{m}^{-2}$. This quasar is at a redshift of $\mathrm{z}=0.536$ so the rest frame RMs are larger by a factor $(1+z)^{2}$ or 2.4 .

The high RMs found in these quasar cores are unlikely to be produced by a cluster environment or by interactions of the jet with a dense environment since both phenomena would favor the production of high RMs in the jet. A more likely explanation is that they are produced by ordered magnetic fields in the narrow line region. Furthermore, the high RM phenomenon is not restricted to CSS sources, but may be found in the cores of typical quasars when they are observed with sufficiently high resolution.

Acknowledgments. The National Radio Astronomy Observatory is a facility of the National Science Foundation, operated under a cooperative agreement by Associated Universities, Inc.

\section{References}

Cawthorne, T. V., et al. 1993. ApJ, 416, 496-518.

Dreher, J. W., Carilli, C. L., \& Perley, R. A. 1987. ApJ, 316, 611-625.

Inoue, M., et al. 1995. PASJ, 47, 725-737.

Taylor, G. B., Inoue, M., \& Tabara, H. 1992. A\&A, 264, 421-427.

Taylor, G. B., \& Perley, R. A. 1993. ApJ, 416, 554-562.

Taylor, G. B., Ge, J.-P., \& O'Dea, C. P. 1995. AJ, 110, 522-528.

Udomprasert, P. S., et al. 1997. ApJ, 483, L9-12. 